\title{
Dissecting Abdominal Aortic Aneurysm
}

National Cancer Institute

\section{Source}

National Cancer Institute. Dissecting Abdominal Aortic Aneurysm. NCI Thesaurus. Code C27085.

A dissecting aneurysm in the portion of the descending aortic wall that is within the abdomen. 